\section{Multiaxial Fatigue Spectrum Editing by Using Combined Wavelet Analysis and Stress Invariant Approach}

\author{
Anahita Imanian ${ }^{1, *}$, Kelvin Leung $^{1}$, and Nagaraja Iyyer ${ }^{1}$ \\ ${ }^{1}$ Technical Data Analysis, Inc., 3190 Fairview Park Dr, Suite \\ 650, Falls Church, Virginia, 22042, USA
}

\begin{abstract}
The practicalities of structural fatigue testing limit the fidelity of the cyclic load history that can be applied to a test structure. Testing is, therefore, a compromise between fatigue damage fidelity and test economy. A new methodology is proposed for multiaxial loading spectrum editing to extract cycles that contribute negligible damage during fatigue crack initiation. The method is based on projection by projection $(\mathrm{PbP})$ technique and wavelet transform analysis (WTA) procedure. In this approach, the cycles with negligible contribution to damage in every decoupled projected loading path (i.e. obtained from $\mathrm{PbP}$ approach) are extracted using the WTA procedure. Each extracted segment is then replaced with an equivalent cycle that produces the same amount of damage. The effectiveness of the edited spectrums is evaluated by the degree of fatigue damage retention as the original damage and preservation of statistical parameter values.

As a case study, the proposed approach has been applied to the numerically produced random bending-torsion fatigue spectrum in plane-stress condition. The result shows an average of $75 \%$ reduction of the original spectrums with retention of $90 \%$ of the original spectrums' damage values.
\end{abstract}

\section{Introduction}

The service load histories corresponding to fatiguecritical locations on engineering structures can contain extremely large numbers of time points in a single record. Employing these large histories in either analysis or testing can be very time-consuming and therefore very expensive. These challenges could become even more critical when dealing with multi-axial loading spectrums. The accurate development of a representative load spectrum is vital when testing the fatigue performance of a structure. The challenge is, therefore, how to reduce the density of load cycles for test purposes while maintaining fatigue performance accuracy and fidelity, i.e. spectrum editing.

Spectrum editing methods identify and remove the sections of the load history that are relatively insignificant for damage summation, in order to reduce subsequent analysis and testing costs. Various spectrumediting methods have been developed and studied by fatigue researchers especially in aircraft and ground vehicle industries. Among these techniques, omission based editing methods use a gating or filtering algorithm to maintain sequence effects by directly removing cycles from the time sequence [1, 2]. Further, cycles can be removed based on crack opening [3]; load banding (removing loads within a range, often around zero) [4]; or loading time-frequencies $[5,6]$. However, many of these methods challenge a loss of damage content from truncation as a compromise when selecting their truncation level. As a consequence other attempts formed to return damage to the spectrum, e.g. load scaling [7] or, using the principle of equivalent damage [8].

Many structural component or parts are subject to multiaxial loading conditions such as rotorcraft structure where the complex unsteady combination of the dynamic load with vibratory response causes a significant degree of multiaxiality. Current spectrum editing methods were widely examined for scaler valued load spectrums, and to the understating of the author, there is no method available to address multiaxial fatigue spectrum editing problem. This paper proposes a new method to edit the multiaxial fatigue spectrum for high-cycle fatigue loading. The proposed approach is based on the combination of the time-frequency signal analysis procedure, and stress invariant based multiaxial fatigue criterion for fatigue crack initiation damage. The timefrequency signal analysis method is based on WTA which enables to characterize the nonstationary and transitory behavior of random signals and to identify cycles with negligible damage contributions while keeping the sequence of a spectrum. Stress invariant which has been defined as a PbP approach decouples the loading path along fixed directions of a principal reference frame in the deviatory space [2], and define nonlinear cumulative damage criteria based on the damage evaluated at each projection. In the proposed approach, the wavelet transform analysis procedure applies on every projected loading path (i.e. obtained from $\mathrm{PbP}$ approach) to identify the segments with negligible damage contributions. Each extracted segment then is replaced with a scaled cycle that produces the equivalent amount of damage.

The remainder of this paper is organized as follows: in Section 2, we provide a detailed description of WTA. Section 3 presents the PbP damage criterion approach. Section 4 explains the wavelet-based editing algorithm and damage equivalent method for replacing extracted segments which have negligible damage contributions to the total damage. In Section 5, the proposed approach is applied to a 2-D fatigue spectrum, followed by conclusions.

\section{Wavelet transform analysis}

Most fatigue histories exhibit nonstationary characteristics and need time-frequency analysis. Timefrequency methods such as short time Fourier transform (STFT) and Winger-Ville distribution use the same window for the analysis of the entire spectrum, and are unable to analyse spectrums with rapid and slow transient fatigue spectra. The wavelet transform on the other hand uses a different window function for analyzing different frequency band over time, and 
therefore enables to capture non-stationary features of the spectrums by decomposing the signals into their frequency bands.

The wavelet transform method [9] provides varying time and frequency resolutions by using windows with different lengths. To solve the resolution problem, this technique allows the use of long time window (more precise low-frequency information) and shorter windows (high-frequency information). The wavelet transform calculates a similarity index between the signal being analyzed and the wavelet, called the coefficient. It is the result of the regression of an original signal produced at different scales and different sections on the wavelet. If the index is large, the resemblance is strong otherwise, it is slight. Generally, the wavelet coefficient, $C$, is expressed with the following integral:

$$
C(a, b)=\int_{-\infty}^{\infty} x(t) \psi_{a, b}(t) d t
$$

where $x(t)$ is the spectrum/signal to be analysed, $\psi_{a, b}$ is the mother wavelet, parameter $a$ represents the scale factor which is a reciprocal of frequency, the parameter $b$ indicates the time shifting or translation factor, and $t$ is time. The wavelet transform first decompose the spectrum into frequency bands, and then analysing them in time as:

$$
C W T(a, b)=\int_{-\infty}^{\infty} x(t) \frac{1}{\sqrt{(a)}} \psi\left(\frac{t-b}{a}\right) d t
$$

and

$$
x(t)=\frac{1}{C_{\psi}} \int_{0}^{\infty} \int_{-\infty}^{\infty} C W T(a, b) \psi\left(\frac{t-b}{a}\right) \frac{d a d b}{a^{2}}
$$

where $C_{\psi}$ is the constant that depends on $\psi$, and $C W T(a, b)$ is the continuous wavelet transform of $x(t)$. The wavelet coefficient indicates how the energy in the signal is distributed in the time-frequency plane.

In this paper, we choose digital wavelet transform (DWT) technique due to its computational efficiency. DWT was first introduced by Daubachies [10]. He realized that by choosing special orthogonal wavelet bases the infinitely redundant CWT can be critically sampled on a dyadic grid. DWT can be computed very efficiently by using techniques from the theory of filter banks. In this method, the DWT of a signal, $x(t)$, is calculated by passing it through a series of filters as shown in Fig. 1. First, the samples are passed through a low pass filter $\mathrm{g}$. The signal is also decomposed simultaneously using a high pass filter $h$. The outputs include the detail coefficients (from the high-pass filter) and approximation coefficients (from the low-pass). This decomposition is repeated to further increase the frequency resolution and the approximation coefficients decomposed with high and low pass filters and then down-sampled. Fig. 1 shows an example of a three- level filter bank.

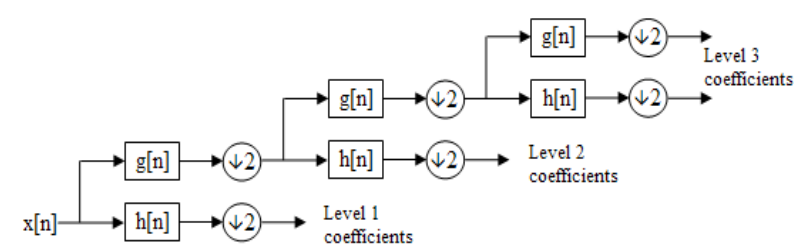

Fig. 1. Three level filter bank.

\section{Projection by projection damage criteria}

Projection by projection $(\mathrm{PbP})$ method is a type of stress invariant based approaches usually employed in the medium/high-cycle fatigue regimes [11]. The method has shown to provide accurate fatigue damage estimates for variable amplitude multiaxial loading spectrums [12]. This method computes fatigue damage by decoupling the loading path, $\Omega$, along the axes of a principle reference frame in the deviatory space, which are located by the eigenvectors, $\mathbf{U}$, of the covariance matrix, $\mathrm{C}^{\prime}$, of the deviatory stress vector, $\boldsymbol{s}(t)$. The elements of the matrix $\mathrm{C}^{\prime}$ are:

$$
\begin{aligned}
c_{h k} & =\operatorname{cov}\left(s_{h}(t), s_{k}(t)\right) \\
& =E\left[\left(s_{h}(t)-s_{m, h}(t)\right)\left(s_{k}(t)-s_{m, k}(t)\right)\right]
\end{aligned}
$$

where $s_{m, h}$ and $s_{m, k}$ are, respectively, the coordinates $h$ and $k$ of the centroid of the path $\Omega$. This matrix has five eigenvalues and five orthogonal eigenvectors for sixdimensional space stress process, $\boldsymbol{\sigma}(t)$. The eigenvalues are the principal moments of inertia, whereas the eigenvectors are the principal directions of the stress tensor path calculated with respect to the centroid itself. For a multiaxial variable amplitude loading spectrum, each projected loading path along the principal direction, $\Omega_{p, i}(t)$, is a uniaxial variable amplitude process. Fatigue cycles in the $i_{\text {th }}$ projected load path, $\Omega_{p, i}(t)$, are identified by rainflow method while accounts for both the amplitude, $\left(\sqrt{J_{2 a}}\right)_{i j}$, of the deviatoric stress, in each counted cycle (identified by $j$ ), and the maximum of hydrostatic stress, $\sigma_{H \max } i j(t)$. Here, $\sqrt{J_{2 a}}$ is the square root of the amplitude of second invariant of stress deviator.

Fatigue damage for every projected loading path is evaluated based on a reference fatigue strength curve in a modified Wohler diagram, see Fig. 2. 


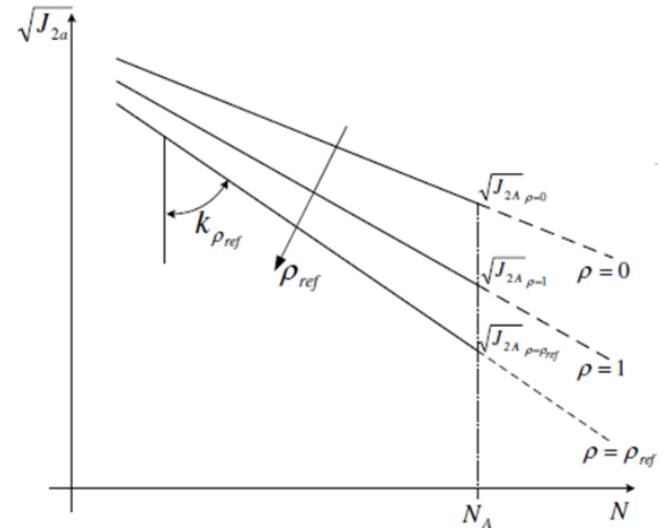

Fig. 2. Modified log- $\log$ Wohler diagram for the stress invariant based approach which shows the SN curves for axial $(\rho=1)$, torsion $(\rho=0)$ and general multiaxial stress $\left(\rho=\rho_{\text {ref }}\right)$. (Reprinted from [11]).

The reference curve for an arbitrary multiaxial loading history is located by a reference stress ratio as:

$$
\rho_{\text {ref }}=\sqrt{3} \frac{\overline{\sigma_{H, \max }}}{\sqrt{\sum_{i} J_{2 a_{\text {iref }}}}}
$$

Reference stress ratio, $\rho_{\text {ref }}$, quantifies the proportionality degree of multiaxial stress in terms of the mean value of the maximum hydrostatic pressure $\overline{\sigma_{H, \max }}=$ $\operatorname{mean}_{i j}\left(\sigma_{H \max i j}\right)$ and the mean amplitude $J_{2 a_{\text {iref }}}=$ $\left.\operatorname{mean}_{j}\left(\sqrt{J_{2 a}}\right)_{i j}\right)^{2}$ of all cycle counted in the $i_{\text {th }}$ projected loading path, $\Omega_{p, i}(t)$. A value $\rho=1$ represents pure axial loading, while $\rho=0$ indicates pure torsion loading.

The calculated $\rho_{\text {ref }}$ value allows to estimate the reference fatigue amplitude strength, $\sqrt{J_{2 A}} \rho_{\text {ref }}$, and the inverse slope $k_{\text {ref }}$ of the reference SN curve by a linear interpolation relations [11] as:

$$
{\sqrt{J_{2 A}}}_{\rho_{\text {ref }}}={\sqrt{J_{2 A}}}_{\rho=0}+\rho_{\text {ref }}\left({\sqrt{J_{2 A}}}_{\rho=1}-{\sqrt{J_{2 A}}}_{\rho=0}\right)
$$

$$
k_{\text {ref }}=k_{\rho=0}+\rho_{\text {ref }}\left(k_{\rho=1}-k_{\rho=0}\right)
$$

where ${\sqrt{J_{2 A}}}_{\rho=1}$ and ${\sqrt{J_{2 A}}}_{\rho=0}$ are the fatigue limits, expressed in terms of amplitude of the square root of the second invariant of the stress deviator, under fullyreversed uniaxial and torsional loading, respectively, and $k_{\rho=1}$ and $k_{\rho=0}$ are the corresponding inverse slopes of the Wohler curves. Typically, $N_{A}$ is considered as $2 \times 10^{6}$ cycles.

Using the above formulations, fatigue damage, $D_{i j}$, due to each identified cycle, $j$, can be estimated through the following expression:

$$
D_{i j}=\left(\frac{\sqrt{J_{2 a}}{ }_{i j}}{\sqrt{J_{2 A}} \rho_{\text {ref }}}\right)^{k_{r e f}} \cdot \frac{1}{N_{A}}
$$

The total damage relative to the $i_{\text {th }}$ projection can then be obtained by Palmgren-Miner linear accumulation rule [12] as follows:

$$
D\left(\Omega_{p, i}\right)=\sum_{j} D_{i j}
$$

A nonlinear accumulation rule finally can be applied to the damage of every projected loading path to obtain the damage for the complete loading path $\Omega$ as shown by Eq. 9. This rule was acknowledged by many authors [11, $13]$.

$$
D(\Omega)=\left[\sum_{i}\left(D\left(\Omega_{p, i}\right)\right)^{\frac{2}{k_{r e f}}}\right]^{\frac{k_{r e f}}{2}}
$$

\section{Wavelet transform and equivalent damage methods for fatigue data editing}

The fatigue spectrum editing algorithm uses the wavelet coefficient amplitude as a parameter to determine the threshold value for the low damage cycles identification process. The wavelet coefficient amplitudes are obtained from DWT of each projected loading history, $\Omega_{p, i}, i=1,3$. The extraction processes is performed by searching segments with maximum amplitude less than the specific threshold value over both time history and decomposed levels. After all segments with low contribution to damage are identified, the process of replacing each segment with a cycle with an equivalent damage value is performed in order to compensate for each segment's damage. In this process the equivalent stress amplitude is obtained by the following expression for $i=1,3$ :

${\sqrt{J_{2 a}}}_{e q, z}={\sqrt{J_{2 A}}}_{\rho_{\text {ref }}}\left(N_{A} \cdot D_{z}\left(\Omega_{p, i}\right)\right)^{1 / k_{\text {ref }}}$

where $D_{z}\left(\Omega_{p, i}\right)$ is the damage produced at $z_{\text {th }}$ extracted segment that can be obtained by Eq. 7 .

Once the extracted segments are identified and replaced with an equivalent cycle, the statistical features (e.g. root mean square (rms) and kurtosis) of the edited signal will be compared with the original signal. Statistically, the rms is used to quantify the overall energy content of the oscillatory signal and the kurtosis is used as a measure of non-Gaussianity. Mathematically, the rms and kurtosis are defined by the following equations:

$$
r m s=\left(\frac{1}{n} \sum_{i=1}^{n} x_{i}^{2}\right)^{1 / 2}
$$

Kurtosis $=\frac{1}{n(r m s)^{4}} \sum_{i=1}^{n}\left(x_{i}-\bar{x}\right)^{4}$

where $x_{i}$ is the instantaneous value, $n$ is the number of points, and $\bar{x}$ is the mean of the time history. In each projected axis, the edited spectrum replicates the spectrum statistical parameter and fatigue damaging characteristics of the original time history. The optimum threshold values are accordingly determined and it is based on the effectiveness of retaining the characteristics of the original spectrums. 


\section{Case study for bending-torsion fatigue history}

The discussed wavelet base editing algorithm was applied on an out-of-phase random amplitude bendingtorsion fatigue history and for the plane-stress condition. Under bending-torsion condition, the time histories of the random axial stress, $\sigma_{x x}(t)$, and the random shear stress, $\sigma_{x y}(t)$, are numerically produced using following expressions:

$$
\begin{aligned}
& \sigma_{x x}(t)=\sigma_{x x, m}+\sum_{i=1}^{400} \sigma_{x x, a} \sin \left(2 \pi \omega_{i} t\right) \\
& \sigma_{x y}(t)=\sigma_{x y, m}+\sum_{i=1}^{400} \sigma_{x y, a} \sin \left(2 \pi \omega_{i} t-\frac{\pi}{3}\right)
\end{aligned}
$$

where the suffix $m$ identifies the mean value of the spectrums, $\sigma_{x x, m}=300 \mathrm{MPa}$, the frequency is $\omega_{i}=$ $300 \times \operatorname{Normal}(0,1), i=1,2, \ldots, 400, \quad \sigma_{x y, m}=$ $200 M P a$, the suffix $a$ is the amplitudes, $\sigma_{x x, a}=3 M P a$, $\sigma_{x y, a}=2 M P$, and $\frac{\pi}{3}$ is the phase shift between the applied stress components. It should be noted that the random nature of the frequency in the sinusoidal terms lead to the randomness of amplitudes as well (Please see Fig. 3).
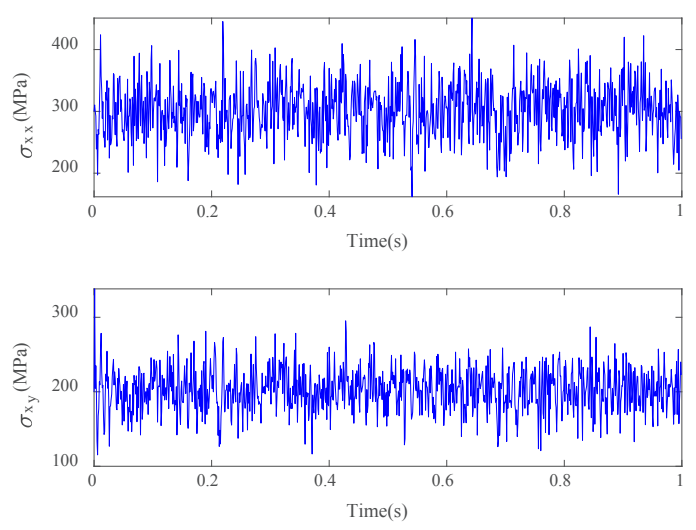

Fig. 3. Random bending-torsion spectrum

The non-null components of the vector associated with the deviatory stresses are:

$$
\begin{aligned}
& s_{1}(t)=\frac{1}{\sqrt{3}} \sigma_{x x}(t) \\
& s_{3}(t)=\sigma_{x y}(t)
\end{aligned}
$$

Fig. 4 shows the random bending-torsion loading path in the two-dimensional deviatory space, the principal reference frame $\left(s_{p, 1}, s_{p, 3}\right)$, and the projected loading paths, $\Omega_{p, i}(t), \mathrm{i}=1,3$.

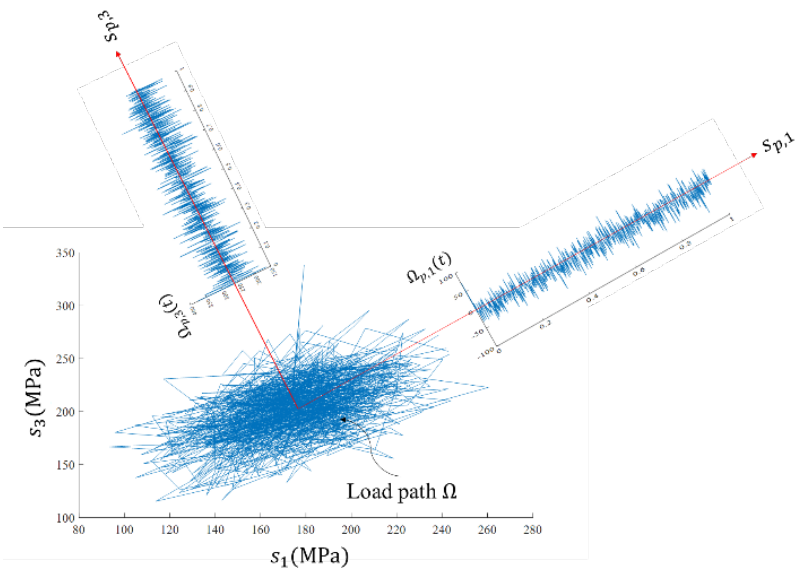

Fig. 4. Bending-torsion random loading path $\Omega$ in the twodimensional deviatory space. The principal reference frame, located by unit vectors $\left(s_{p, 1}, s_{p, 3}\right)$, is used to obtain the stress projections, $\Omega_{p, i}(t), \mathrm{i}=1,3$.

The projected loading elements along the axes of principle reference frame are obtained by projecting the deviatory stresses along the direction of the principal directions. Along these directions, fatigue damage can be expressed as:

$$
D_{i j}=\left(\frac{{\sqrt{J_{2 a}}}_{i j}}{\sqrt{J_{2 A \rho=\rho_{\text {ref }}}}}\right)^{k_{\text {ref }}} \cdot \frac{1}{N_{A}} \quad i=1,3 .
$$

The material which is considered for this study is $\mathrm{Al}$ 6082-T6, where the parameters of the reference SN curve are adapted from [14], and listed in Table 1:

Table 1. Fatigue properties for the considered material.

\begin{tabular}{|c|c|c|c|c|c|}
\hline Material & $\begin{array}{c}\text { Number of } \\
\text { available data }\end{array}$ & $\rho$ & $k_{\rho}$ & $\sqrt{J_{2 A} \rho}$ & $T_{\sigma}$ \\
\hline Al 6082-T6 & 45 & 1 & 6.90 & 75 & 1.07 \\
& & 0 & 7.10 & 77 & 1.40 \\
\hline
\end{tabular}

After projecting the load history along the principle directions the wavelet-base fatigue editing algorithm is applied on every projected loading path, $\Omega_{p, i}(t), \mathrm{i}=1,3$. The wavelet transform algorithm uses $12^{\text {th }}$ order (Daubachies) wavelets as the basis functions. Five decomposition levels are selected for DWT of each projected loading history, $\Omega_{p, i}, \mathrm{i}=1,3$. For example, Fig. 5 and 6 depict the decomposition levels for $\Omega_{p, 1}$ and $\Omega_{p, 2}$, respectively. The wavelet toolbox in MATLAB software was used for DWT.

The low damage contributing cycles identification processes is performed by searching segments with the maximum amplitude less than the threshold values 35 and 60 for $\Omega_{p, 1}(t)$ and $\Omega_{p, 3}(t)$, respectively, over both time history and decomposed levels. The extracted segments are replaced with an equivalent cycle that produces the same amount of damage in order to retain the damage contributed to each extracted segment as shown in Fig. 7 (a) and Fig. 7(b). Furthermore, the effectiveness of the extracted and replaced segments is 
evaluated by the preservation of statistical parameter values (kurtosis and rms) for every projected loading, $\Omega_{p, 1}(t)$ and $\Omega_{p, 3}(t)$, where the difference of $\pm 15 \%$ in the rms and kurtosis values was used.
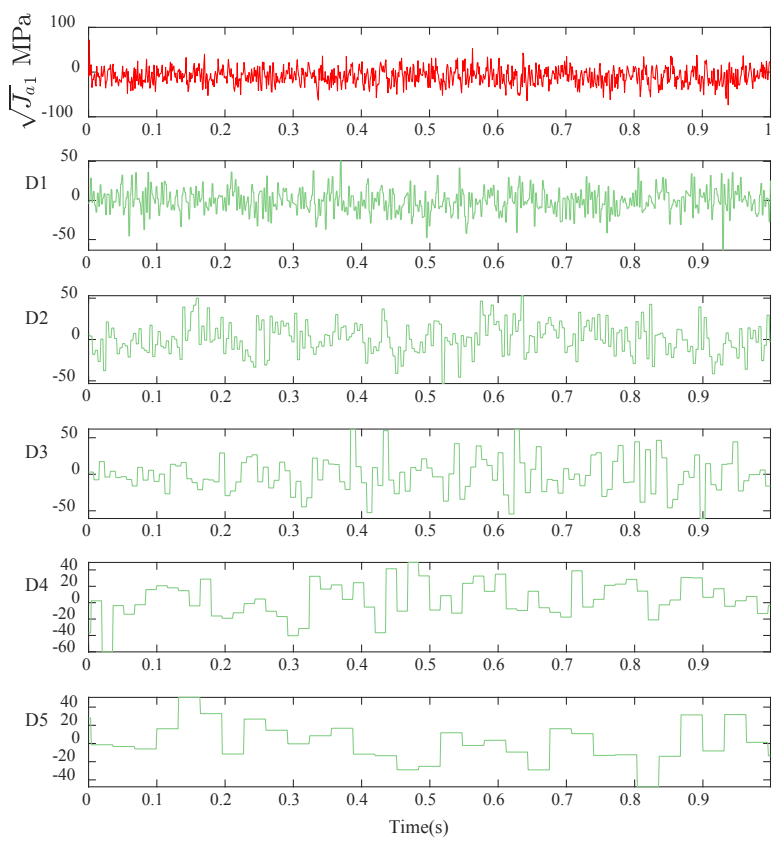

Fig. 5. Wavelet decomposition levels for projected loading path $\Omega_{p, 1}$.
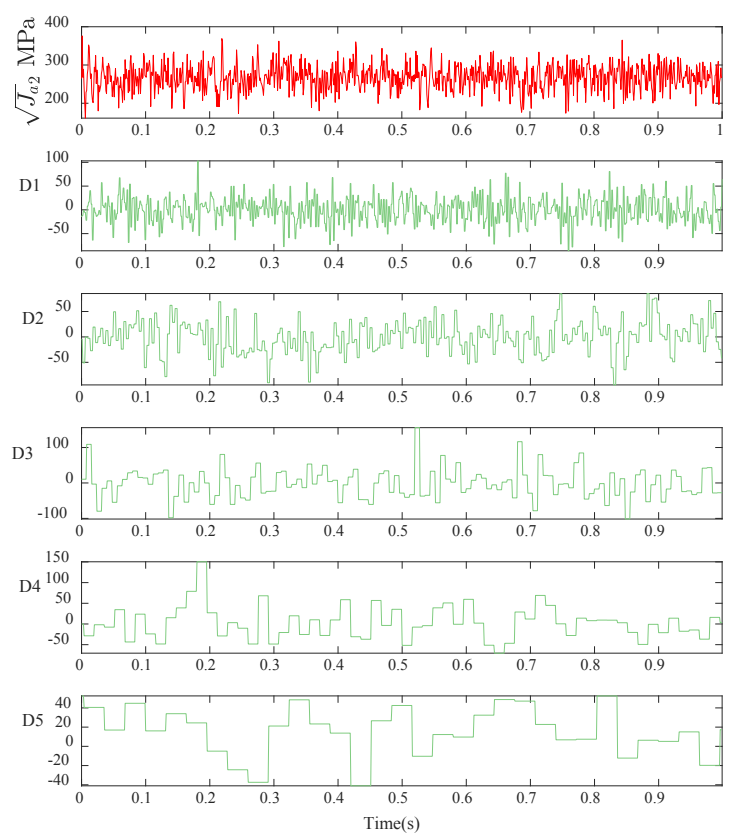

Fig. 6. Wavelet decomposition levels for projected loading path $\Omega_{p, 3}$

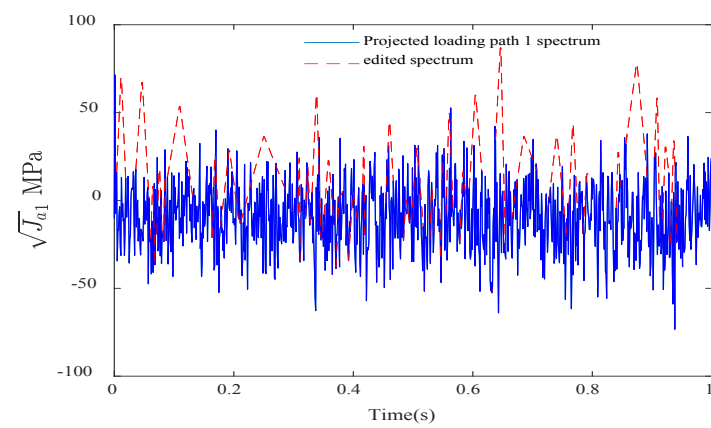

(a)

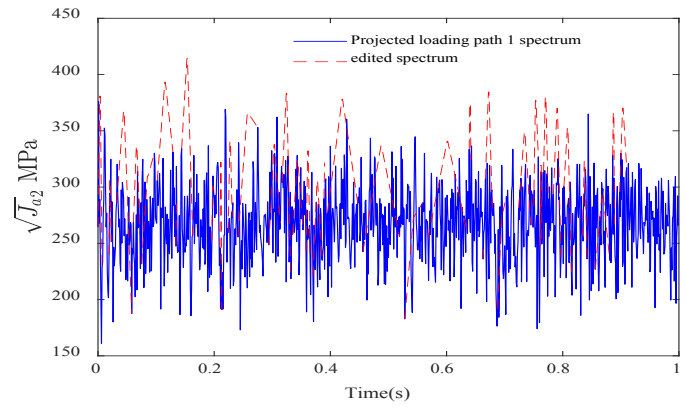

(b)

Fig. 7. Replacement of segments with low contribution to damage with a cycle producing an equivalent damage, (a) for projected loading path, $\Omega_{p, 1}$, and (b) for projected loading path, $\Omega_{p, 3}$. Blue line depicts the original projected loading path spectrum and red dash line shows the edited spectrum.

The results show that the spectrums in the projected loading paths, $\Omega_{p, 1}(t)$ and $\Omega_{p, 2}(t)$ are shorted by $73 \%$ and $77 \%$, respectively. The error of damage between original projected loadings spectrums $\Omega_{p, 1}(t)$ and $\Omega_{p, 2}(t)$, and edited spectrums are obtained as $-8 \%$ and $7.7 \%$, respectively. The total damage error between the original and edited loading spectrum in the principle frame is $-7 \%$. The edited load spectrum in each projected axis can finally back-projected to the original frame by using Eq. (15) and (16).

\section{Conclusions}

This paper discussed the study of multiaxial fatigue spectrum editing by using the time-frequency wavelet analysis approach and $\mathrm{PbP}$ damage criteria method. The newly proposed method allowed for identification of cycles with low contribution to damage in the decoupled loading axes obtained by $\mathrm{PbP}$ method. An equivalent damage method was then used to compensate for the damage associated with extracted segments. The numerically produced bending-torsion fatigue history was used to apply the proposed approach. The results of the spectrum editing showed that, on average, $75 \%$ of the spectrum lengths in the first and second axes are reduced with the retention of $90 \%$ of the original fatigue damage. 
Spectrum editing with the proposed approach can accelerate the fatigue test by at least a factor of four to achieve the same or similar fatigue crack initiation damage. The proposed method for multiaxial loading spectrum is entirely new, and the level of acceleration it provides is anticipated to have a significant impact on reducing the costs of testing and provide beneficial solutions not otherwise possible. Further work is planned to systematically investigate the application of the technique for six-dimensional load spectra, evaluation of the proposed approach for real fatigue history spectrums with different material and geometries, and accounting for crack growth damage.

\section{References}

[1] A. Lanciotti and L. Lazzeri, Int. J. Fatigue, 14 (1992) [2] P. R. Stephens, P. M. Dindinger, and J. E. Gunger, Int. J. Fatigue, 19 (1997)

[3] R. Sunder, Int. J. Fatigue, 7 (1985)

[4] R. L. Hewitt, J. P. Weiss, and P. K. Nor, ASTM STP 1439, (2005)

[5] C. S. Oh, Int. J. Fatigue, 23 (2000)

[6] Abdullah. S, J. Eng. Appl. Sci. 2 (2007)

[7] M. McCoy and R. Ogden, Defence Sci. Tech. Org., (2009)

[8] H. J. Grover, Fatigue of Aircraft Structures, (1966)

[9] S. Berry, IEEE, pp. 653-659, (1999)

[10] I. Daubechies, Comm. Pure. Appl. Math, 41(1988)

[11] A. Cristofori, L. Susmel, R. Tovo, Int. J. Fatigue, 30 (2008)

[12] A. Fatemi, L. Yang, Int. J. Fatigue, 20 (1998)

[13] A. Cristofori, A New Perspective in Multiaxial Fatigue Damage Estimation, Ph.D. thesis, (2007)

[14] L. Susmel L, N. Petrone, Biaxial/ Multiaxial Fatigue Fract. pp. 83-104, (2003) 